\title{
The nature of Hen 3-1312: A post-AGB star in a binary system ${ }^{\star} \star \star$
}

\author{
C. B. Pereira ${ }^{\star \star \star}$ \\ Observatório Nacional, Rua José Cristino, 77, CEP 20921-400, São Cristóvão, Rio de Janeiro-RJ, Brazil
}

Received 29 April 2003 / Accepted 8 August 2003

\begin{abstract}
This work reports the low- and high-resolution spectroscopic diagnostic diagrams, radial velocity, stellar parameters and abundance analysis of the planetary nebula Hen 3-1312. The low- and high-resolution spectra reveal that Hen 3-1312 is in fact a very-low-excitation object, in a binary system with a supergiant as a cool central star. The analysis of the high-resolution spectrum shows the cool stellar component to have an effective temperature of $T_{\text {eff }}=6500 \pm 100 \mathrm{~K}$ and a surface gravity of $\log g=0.8 \pm 0.2$ corresponding to a spectral type of $\mathrm{F}(6-7) \mathrm{I}$. These parameters result in an estimated primary luminosity of $4100 L_{\odot}$, implying a distance of $4400 \mathrm{pc}$ which is in agreement with previous determinations. The abundance analysis reveals Hen 3-1312 to be a metal-poor object having $[\mathrm{Fe} / \mathrm{H}]=-1.1$. The mean abundances of carbon, nitrogen and oxygen are found to be solar, however the $\alpha$-elements (Mg, Si and Ca) are underabundant relative to the Sun. The abundance profile of Hen 3-1312 is analyzed and compared with other classes of stars with similar atmospheric parameters.
\end{abstract}

Key words. stars: abundances - stars: binaries: symbiotic - stars: AGB and post-AGB

\section{Introduction}

Hen 3-1312 was first discovered as emission-line object by Sanduleak \& Stephenson (1972), bearing the name SaSt 2-12 as a very-low-excitation compact nebula, e.g. those nebulae that have the $[\mathrm{O}$ II $] 3727$ line comparable or even stronger than $\mathrm{H} \beta$. As it shown in Fig. 1, the central of Hen 3-1312 has a F-G spectral type. Classified by Kohoutek (1978) as proto planetary-nebulae, Hen 3-1312 entered in Acker's (1992) catalogue with a question about its spectral type, F-G? Hen 3-1312 was classified as compact nebula by Lamers et al. (1998).

This work will analyze the low and high resolution spectra of Hen 3-1312 in order to discuss and understand the evolutionary status of this peculiar object. The low resolution data will be used to probe the physical conditions of the nebulae and to do a tentative classification using the diagnostic diagram from Baldwin et al. (1981). The high resolution data will be used to investigate the evolutionary status of the central cool star. The the low-resolution data will be used to measure the fluxes, to determine the reddening and the electronic density. The highresolution data will be used to confirm the previous radial velocity determination from absorption and emission lines and to determine the chemical abundances.

* Based on observations made with the $1.52 \mathrm{~m}$ telescope at the European Southern Observatory (La Silla, Chile) under the agreement with the CNPq-Observatório Nacional (Brazil).

$\star \star$ Table 4 is only available in electronic form at http://www. edpsciences.org

$\star \star \star$ e-mail: claudio@on.br
Table 1. Observation log of Hen 3-1312.

\begin{tabular}{|c|c|c|c|}
\hline Star & Date & Wavelength & $\begin{array}{c}\text { Exp } \\
\text { (s) }\end{array}$ \\
\hline Hen 3-1312 & & $3498 \AA-7423 \AA$ & $\begin{array}{r}900 \\
240 \\
50\end{array}$ \\
\hline
\end{tabular}

\section{Low-resolution observation: The emission spectrum}

\subsection{Observation and reduction}

Spectroscopic observations were performed using a Boller \& Chivens Cassegrain spectrograph at the $1.52 \mathrm{~m}$ ESO telescope of La Silla (Chile). A UV-flooded thinned Loral Lesser CCD \#39 $(2048 \times 2048,15 \mu \mathrm{m} /$ pixel $)$ detector was used giving high quantum efficiency in the blue and in the UV. Observations were taken with the $600 \mathrm{l} / \mathrm{mm}$ grating giving a spectral coverage between $\approx 3500 \AA$ and $7500 \AA$ with a reciprocal dispersion of $1.9 \AA$ pixel $^{-1}$ with a resolution of $4.6 \AA$. Table 1 shows the $\log$ of observations.

The data were reduced to the linear scale, i.e. wavelength versus flux, using IRAF. We followed the standard procedure consisting of bias subtraction, flat-field normalization and wavelength calibration through a He-Ar lamp. Counts were corrected for atmospheric extinction and calibrated for the instrumental chromatic response through observations of standard stars from Oke (1974) and from Hamuy et al. (1994). In the linearized spectra, the line fluxes were measured with the 
Table 2. Observed emission line fluxes of Hen 3-1312 relative to $\mathrm{H} \beta=100$.

\begin{tabular}{|c|c|c|}
\hline$\lambda$ & Ion & Hen 3-1312 \\
\hline 3727 & [O II] & 54.3 \\
\hline 3869 & [Ne III] & 5.5 \\
\hline 3889 & H 8 & 25.5 \\
\hline 3968 & $\begin{array}{c}\mathrm{He} \text { I } \\
{[\mathrm{Ne} \text { III] }} \\
\mathrm{H} \epsilon\end{array}$ & 7.6 \\
\hline 4068 & [S II] & 2.6 \\
\hline 4101 & $\mathrm{H} \delta$ & 19.8 \\
\hline 4340 & $\mathrm{H} \gamma$ & 40.2 \\
\hline 4363 & [O III] & 4.3 \\
\hline 4471 & He I & 2.9 \\
\hline 4861 & $\mathrm{H} \beta$ & 100.0 \\
\hline 4959 & [O III] & 69.3 \\
\hline 5007 & [O III] & 220.9 \\
\hline 5754 & {$[\mathrm{~N} \mathrm{II}]$} & 5.4 \\
\hline 5876 & He I & 15.3 \\
\hline 6300 & [O I $]$ & 3.7 \\
\hline 6312 & [S III] & 2.5 \\
\hline 6363 & [O I] & 1.5 \\
\hline 6548 & {$[\mathrm{~N} \mathrm{II}]$} & 30.6 \\
\hline 6563 & $\mathrm{H} \alpha$ & 420.9 \\
\hline 6584 & [N II] & 91.8 \\
\hline 6678 & He I & 4.5 \\
\hline 6717 & [S II] & 1.0 \\
\hline 6730 & [S II] & 2.3 \\
\hline 7065 & He I & 11.5 \\
\hline 7135 & [Ar III] & 9.1 \\
\hline 7320 & [O II] & 42.7 \\
\hline 7330 & [O II] & 41.9 \\
\hline
\end{tabular}

splot task and blends were resolved using the deblend option. Figure 1 shows the reduced spectra of Hen 3-1312. We estimate the errors in the fluxes to be about $20 \%$ for weaker lines (line fluxes $\approx 10$ on the scale of $\mathrm{H} \beta=100$ ) and about $10 \%$ for stronger lines.

The low-resolution spectrum of Hen 3-1312 shows emission lines of [O III] $] \lambda$ 4959,5007 $\AA$ Balmer lines, [N II] $]$ 6548, $6584 \AA$ and strong [O II] $3727 \AA$ over a continuum of a F-type star. It also shows a strong Ca II absorption confirming the early observations of Sanduleak \& Stephenson (1972) and a later observation of de Freitas Pacheco \& Veliz (1987).

Diagnostic diagrams based on several emission-line ratios have been widely used in astrophysics in order to investigate the different excitation mechanisms (shock-wave heating, photoionization) operating on the gas in different classes of objects such as planetary nebulae, H II Regions, Seyfert galaxies etc. (Baldwin et al. 1981). In this diagram Hen 3-1312 is clearly separated from the planetaries and has the highest $\log (3727 / 5007)$ value when compared with some symbiotic stars.
Table 3. $E(B-V)$ for Hen 3-1312.

\begin{tabular}{lc}
\hline \hline line ratio & Hen 3-1312 \\
\hline $\mathrm{H} \alpha / \mathrm{H} \beta$ & 0.35 \\
$\mathrm{H} \gamma / \mathrm{H} \beta$ & 0.33 \\
$\mathrm{H} \delta / \mathrm{H} \beta$ & 0.42 \\
adopted Balmer decrement & 0.37 \\
\hline$\sigma E(B-V)_{\text {Balmer }}$ & 0.03 \\
\hline
\end{tabular}

\subsection{Extinction}

The extinction will be derived using $\mathrm{H}$ I recombination lines. It was assumed that the reddening law can be represented by the standard interstellar extinction curve $f(\lambda)$ (Osterbrock 1974). For the $\mathrm{HI}$ it was assumed that they are formed under case B conditions (Hummer \& Storey 1987) with a mean electron temperature of $T_{\mathrm{e}}=15000 \mathrm{~K}$ and $N_{\mathrm{e}}=10^{5} \mathrm{~cm}^{-3}$. The extinction parameter was derived from $\mathrm{H} \alpha / \mathrm{H} \beta, \mathrm{H} \gamma / \mathrm{H} \beta$ and $\mathrm{H} \delta / \mathrm{H} \beta$ line ratios. The results of the different reddening calculations are presented in Table 3.

Previuos determinations of Hen 3-1312 was given by Schmeja \& Kimeswenger (2002) which gives 0.39.

\section{High-resolution observation: The absorption spectrum}

\subsection{Observation}

The high-resolution spectrum of Hen 3-1312 analyzed in this work was obtained at the $1.52 \mathrm{~m}$ ESO telescope on February 21, 2000 with the FEROS (Fiberfed Extended Range Optical Spectrograph) echelle spectrograph (Kaufer et al. 1999). The FEROS spectral resolving power is $R=48000$ corresponding to 2.2 pixels of $15 \mu \mathrm{m}$ and the wavelength coverage is from $4000 \AA$ to $9200 \AA$. The nominal $S / N$ ratio was evaluated by the measurement of the rms flux fluctuation in selected continuum windows, and a typical value achieved was $S / N$ 100-150, after $6400 \mathrm{~s}$ of integration time. Figures 2 and 3 show four spectral regions showing absorption and emission lines.

\subsection{Radial velocity}

When Figs. 2 and 3 are examined it is observed that both absorption and emission lines are shifted to shorter wavelengths. Beaulieu et al. (1999) showed that Hen 3-1312 has a radial velocity of $-77 \mathrm{~km} \mathrm{~s}^{-1}$. The fact that both the emission lines and absorption lines are blueshifted it is an important clue that the nebula and the central star are physically associated and not associated by chance. This point is raised because there is another peculiar planetary nebulae candidate with a K-type spectrum suspected to be an optical double and therefore would be not associated with the nebula, M 1-44 (=Hen 2-379) (Lutz \& Kaler 1983). In a spectrum taken with Feros in October 12, 2000 of M 1-44 the emission lines are blueshifted due to a radial velocity of $-100 \mathrm{~km} \mathrm{~s}^{-1}$ (confirming the determination of Beaulieu et al. 1999) but the absorption spectrum does not appear to have the same shift. 


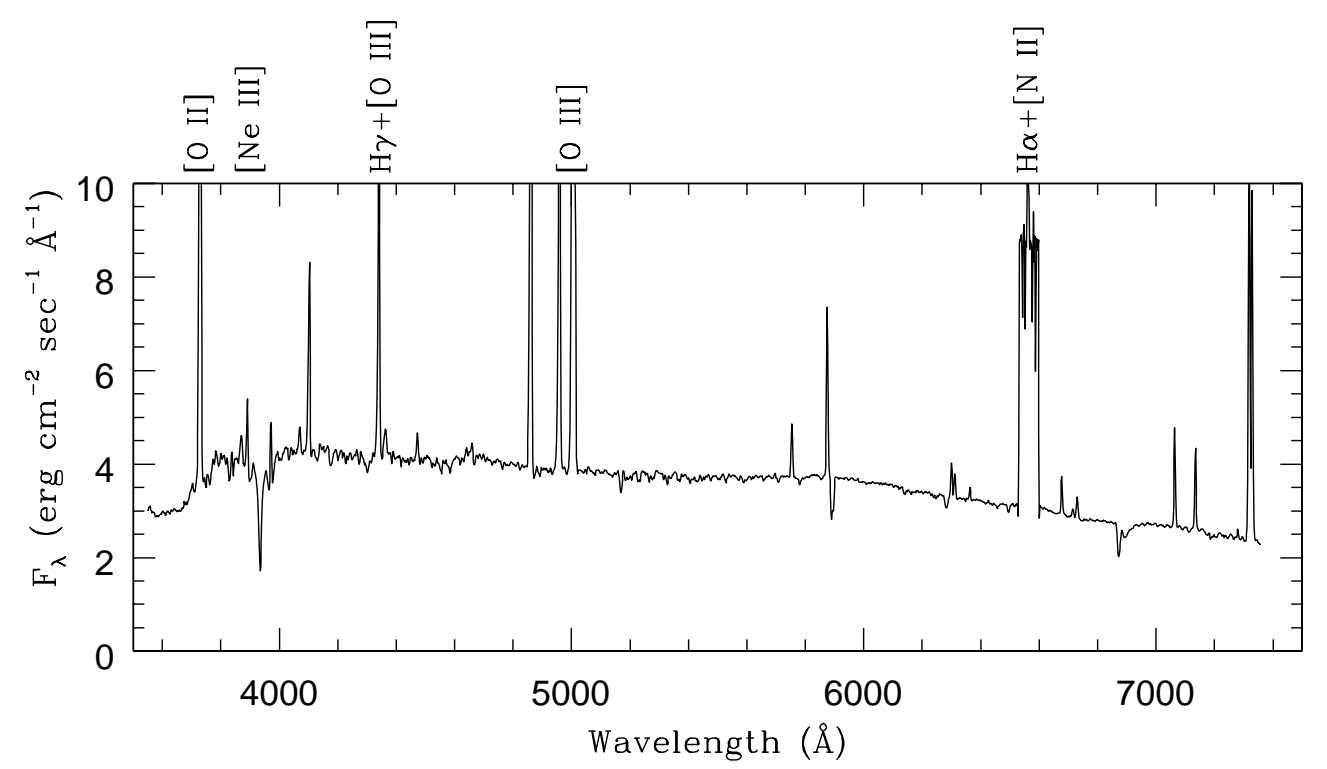

Fig. 1. Optical spectrum of Hen 3-1312. Notice the strength of [O II $] \lambda 3727 \AA$ A. Also notice the presence of a F-type continuum in the spectrum. $\mathrm{H} \alpha$ is saturated.

\subsection{Analysis and results}

\subsubsection{Line selection, measurement and oscillator strengths}

The absorption spectrum of Hen 3-1312, which is responsible for the F-type continuum seen in Fig. 1, shows many atomic absorption lines of Fe I and Fe II as well as transitions due to C I, N I, S I, Ca I, Si I, Mg I, Na I, Zn I and Ba II. We have chosen a set of lines sufficiently unblended to yield reliable abundances. The selected lines are listed in Table 4 for the case of Fe I and Fe II, and in Table 5 for the other elements. In Table 4 we list the $\mathrm{Fe} \mathrm{I}$ and $\mathrm{Fe}$ II lines employed in the analysis and also the lower excitation potential, $\chi(\mathrm{eV})$, of the transitions, the $g f$-values and the measured the equivalent widths. The latter were obtained by fitting Gaussian profiles to the observed ones. The $g f$-values for the $\mathrm{Fe} \mathrm{I}$ and $\mathrm{Fe}$ II lines in Table 4 were taken from Lambert et al. (1996).

\subsubsection{Physical properties of the cool star: Effective temperature and gravity}

The determination of stellar atmospheric parameters, effective temperature $\left(T_{\text {eff }}\right)$, surface gravity $(\log g)$, microturbulence $(\xi)$ and $[\mathrm{Fe} / \mathrm{H}]$ (throughout, we use the notation $[\mathrm{X} / \mathrm{H}]=\log (N(\mathrm{X}) / N(\mathrm{H}))_{\star}-\log \left(N(\mathrm{X}) / N(\mathrm{H})_{\odot}\right)$ are prerequisites for determination of photospheric abundance. The gravity was determined by forcing Fe I and Fe II to yield the same iron abundance at the selected effective temperature. The microturbulent velocity was determined by forcing the abundance determined from individual Fe I lines to show no dependence on equivalent width. The solution for the excitation equilibrium was set to zero slope of the trend between the $\mathrm{Fe}$ I abundances and the excitation potential of the measured lines. The solution thus found is unique, depending only on a set of Fe III lines and the atmospheric model employed, and yields as a byproduct the metallicity of the star $[\mathrm{Fe} / \mathrm{H}]$. The atmospheric parameters were determined in the local-thermodynamicequilibrium (LTE) model atmospheres of Kurucz (1993) using the spectral analysis code MOOG (Sneden 1973). The final adopted atmospheric parameters was $T_{\text {eff }}=(6500 \pm 100 \mathrm{~K})$, $\log g=(0.8 \pm 0.2) \mathrm{cm} / \mathrm{s}^{2}, V_{\mathrm{t}}=(2.6 \pm 0.2) \mathrm{km} \mathrm{s}^{-1}$ and $[\mathrm{Fe} / \mathrm{H}]=-(1.08 \pm 0.14)$. Figure 4 shows the diagrams corresponding, respectively, to the abundances of the individual $\mathrm{Fe} I$ lines plotted against the lower excitation potential and the reduced line strength following the best parameters mentioned above. The internal errors in our adopted effective temperatures $\left(T_{\text {eff }}\right)$ and microturbulent velocity $(\xi)$ can be determined from the uncertainty in the slope of the Fe I abundance versus excitation-potential and $\mathrm{Fe}$ I versus reduced equivalent width $\left(W_{\lambda} / \lambda\right)$ relation. The standard deviation in $\log g$ was set by changing this parameter around the adopted solution until the difference between Fe I and Fe II mean abundance differed by exactly one standard deviation of the $[\mathrm{Fe} \mathrm{I} / \mathrm{H}]$ mean value.

\subsubsection{Abundance analysis}

Abundances of chemical elements of Hen 3-1312 were determined with the local thermodynamic equilibrium (LTE) model atmosphere. The line-synthesis code MOOG was used to carry out the calculations. The results are given in Table 5 where the list of the lines employed for each species and the abundances derived from each line and the ratio $[\mathrm{X} / \mathrm{H}]$ is given. The abundance of oxygen was obtained by spectrum synthesis of the O I triplet at $6156 \AA$. The analysis of these lines gives the final abundance $\log \epsilon(\mathrm{O})=8.63$. The $g f$-values were taken from Venn (1993). Figure 5 shows the synthesis for the oxygen.

The internal scatter in the derived abundances of each element having more than two lines is also given in Table 5. The uncertainties in the derived abundances for the program stars are dominated by three main sources: the $g f$-values, the equivalent widths measurements and the stellar parameters. The uncertainties in the abundances, due to errors in the 

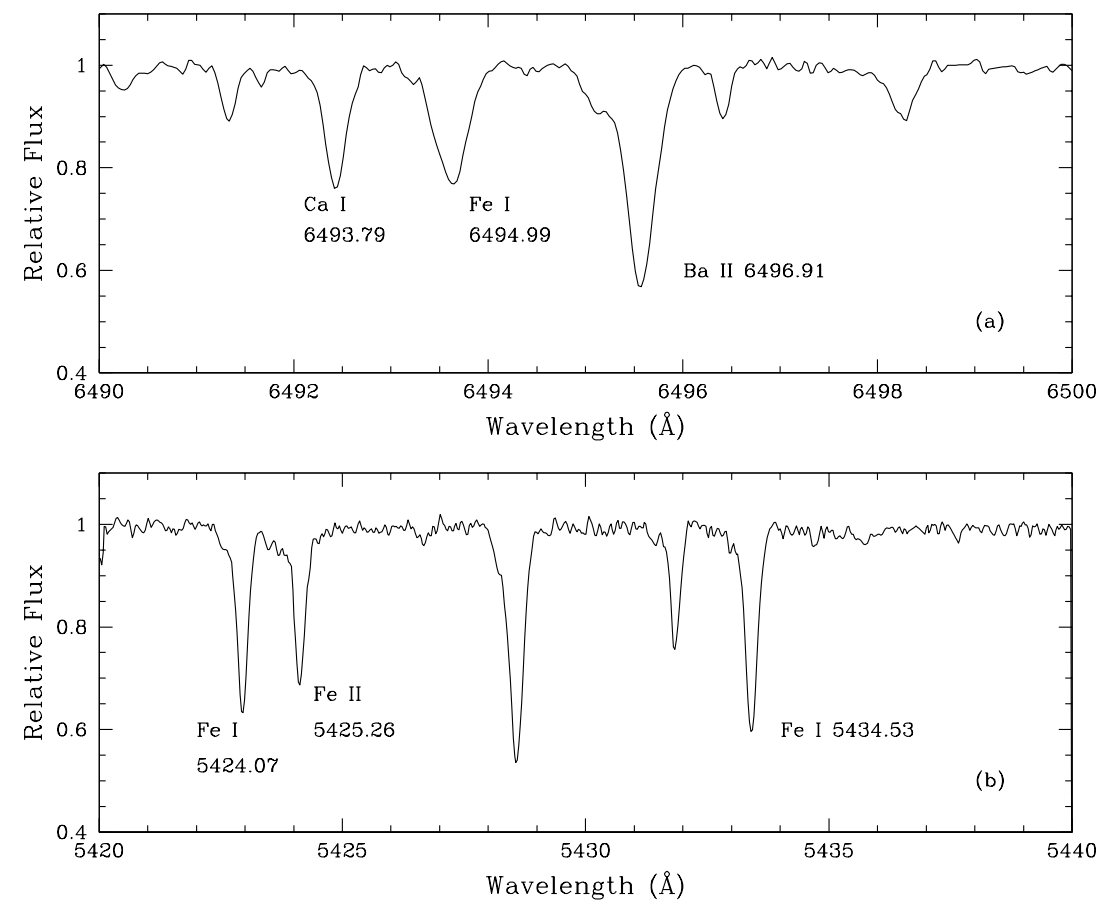

Fig. 2. Observed absorption spectrum of Hen 3-1312 in the neighborhood of Ba II $\lambda 6496$ a) and in the neighborhood of other iron lines b). Notice the blueshifted absorption lines.
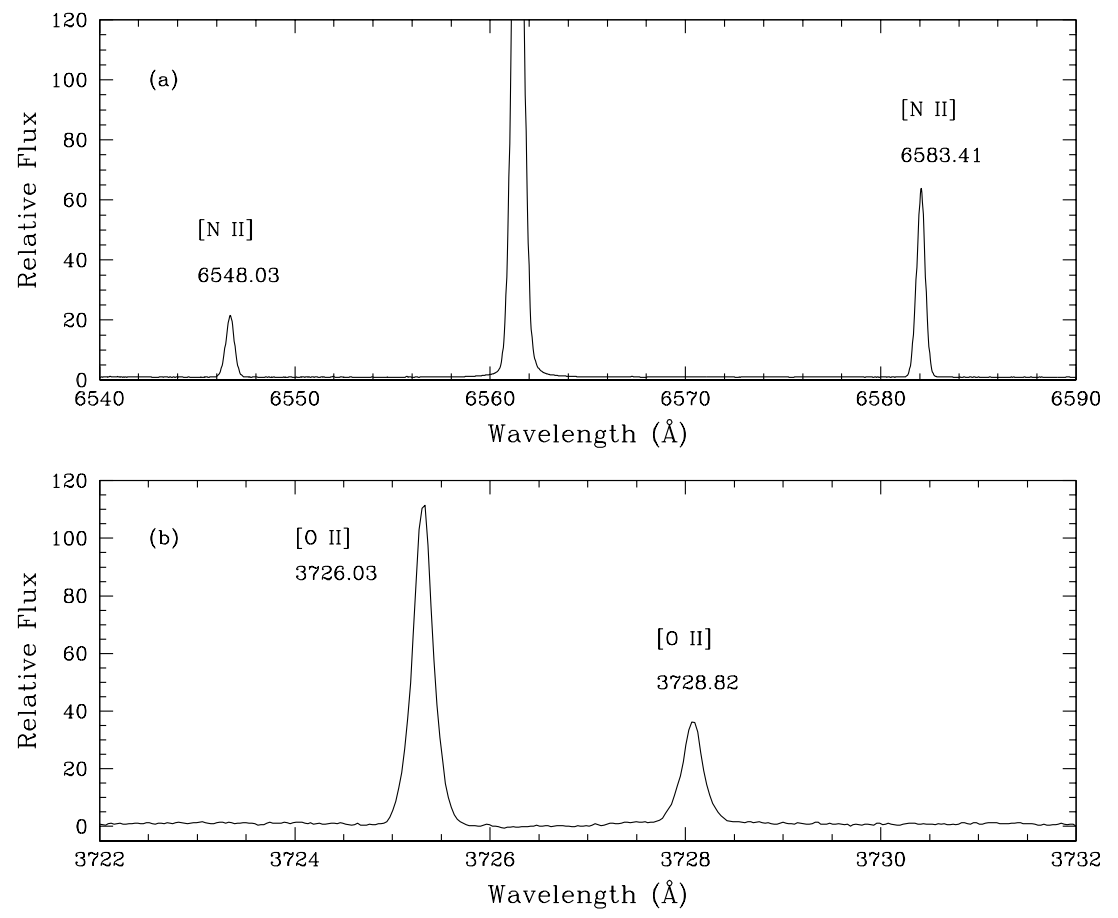

Fig. 3. Observed emission spectrum of Hen 3-1312 in the neighborhood of [N II] lines a) and in the neighborhood of [O II] lines b). Notice the blueshifted emission lines.

stellar atmospheric parameters, were computed by changing these parameters by their standard errors and calculating the changes incurred in the element abundances. The uncertainties due to atmospheric paremeters, $T_{\mathrm{eff}}: \pm 100 \mathrm{~K} ; \log g: \pm 0.2 \mathrm{dex}$ and $V_{\mathrm{t}}: 0.2$ in the derived abundances are: carbon, 0.11 ; nitrogen, 0.58 ; silicon, 0.46 and calcium, 0.25 . For oxygen, an element analyzed via spectrum synthesis, the same technique was used, varying $T_{\text {eff }}, \log g$ and $V_{\mathrm{t}}$, then computing independently the abundance changes introduced by the variation of the above atmospheric parameters. For oxygen, the error in its abundance is 0.23 . 

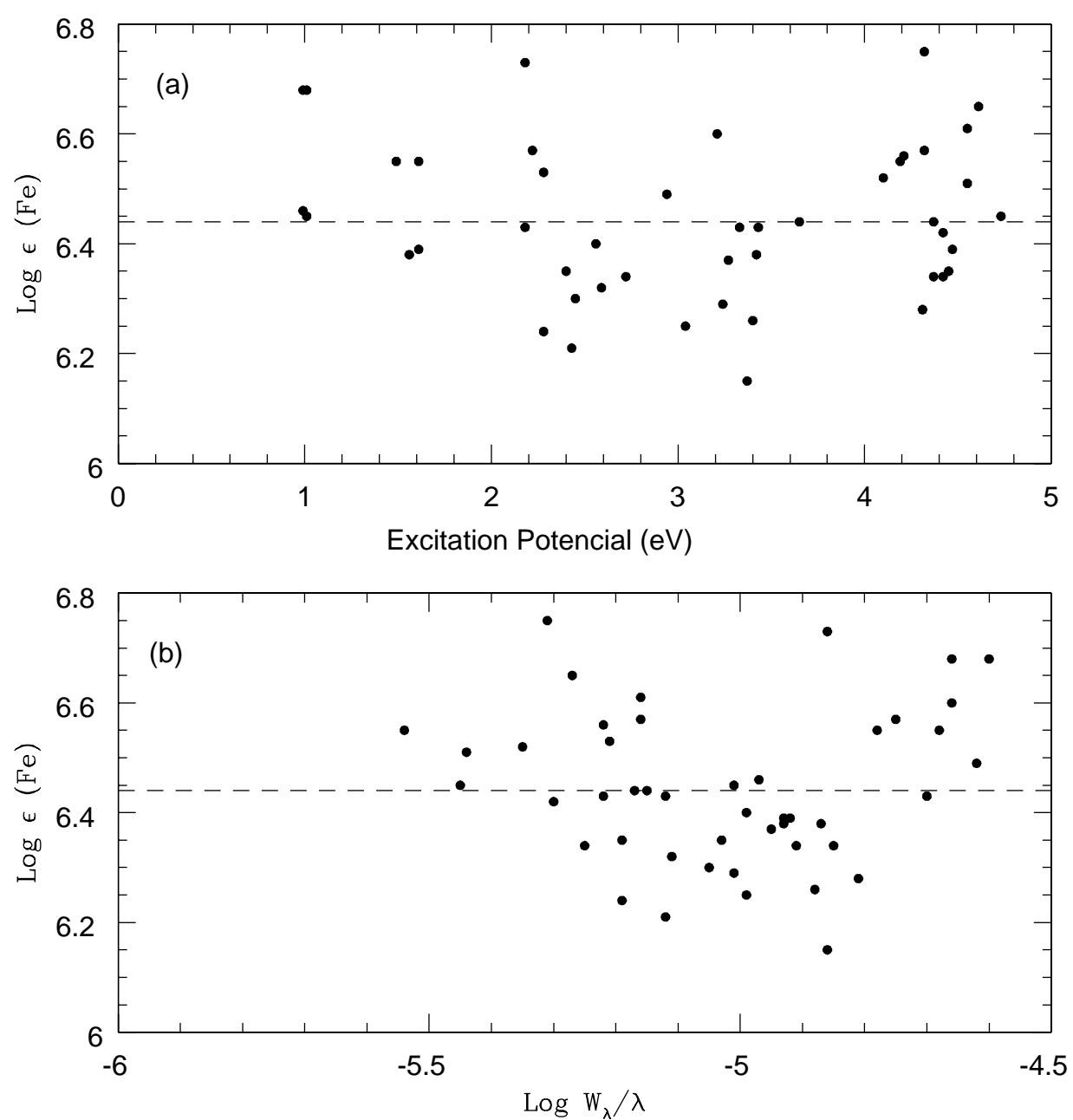

Fig. 4. The abundance of Fe by number $\epsilon(\mathrm{Fe})$ versus excitation potential diagram a) and the abundance of $\mathrm{Fe}$ by number $\epsilon(\mathrm{Fe})$ versus $\log \left(W_{\lambda} / \lambda\right)$ diagram b) for the 58 lines of Fe III employed in the determination of $T_{\text {eff }}, \xi$ and $\epsilon(\mathrm{Fe})$ in Hen 3-1312. The calculation was done with the adopted model $T_{\text {eff }}=6500 \mathrm{~K}, \log g=0.8, \epsilon(\mathrm{Fe})=6.44$ and $\xi=2.6 \mathrm{~km} \mathrm{~s}^{-1}$. The absence of any trend of the individual abundance with excitation potential and microturbulence shows that a correct value for $T_{\text {eff }}$ and microturbulence velocity was chosen.

\section{Discussion}

\subsection{Stellar parameters and evolutionary tracks}

With the derived stellar parameters of $T_{\text {eff }}=6500$ and $\log g=0.8$, the cool component of Hen 3-1312 can be located in $\log g$ - $\log T$ plane and compared to post-horizontal branch evolutionary tracks of both Schönberner (1983) and Blöcker \& Schönberner (1990). These are indicated in Fig. 6 as solid lines for $0.546 M_{\odot}, 0.565 M_{\odot}$ and $0.605 M_{\odot}$. The position of other post-AGB stars already studied is also given in Fig. 6 . The parameters derived here suggest that the cool component in the binary system of Hen 3-1312 consists of a star with $M \sim 0.55 M_{\odot}$. The values of mass, gravity and temperature can be used to estimate a luminosity of $\sim 4100 L_{\odot}$ or $M_{V}=-4.4$. Taking $V=10.0$ (Parthasarathy et al. 2000) and $A_{V}=1.2$ (Sect. 2.2) a distance of $\sim 4400 \mathrm{pc}$ is derived. This distance is in good agreement with the $4040 \mathrm{pc}$ given by Phillips (2001).

\subsection{The abundance pattern}

Since the stellar parameters indicates that the cool component is a supergiant and its position in the $\log g$ - $\log T$ plane is coincident with other post-AGB stars and also displays similar atmospheric parameters of Population I supergiants, its abundance pattern will be compared with these objects. Table 6 gives the mean abundances of some elements and metallicity for Population I supergiants (Luck \& Bond 1989; Luck \& Lambert 1985); post-AGB stars which display the $21 \mu \mathrm{m}$ circunstellar feature (van Winckel \& Reyniers 2000); metal-poor binary post-AGB stars (Trams et al. 1993) and another group of post-AGB stars which are not s-process enriched (Luck et al. 1990; van Winckel 1997). Luck (1993) divided post-AGB stars in two groups, A and B. Those that belong to Group A stars all have [element $/ \mathrm{Fe}$ ] much higher than unity, which reflect that the ratio $[\mathrm{Fe} / \mathrm{H}]$ is not primordial but is a result of grain condensation (Trams et al. 1993) and are members of binary systems. Those that belong to Group B stars are post-AGB stars that do not show extreme iron deficiencies and do not show a large overabundance of carbon. Table 6 is divided into two parts, in the first one the abundances are given in the notation in the scale of $\log H=12.0$ in order to avoid the comparison the [element/Fe], specially for the metal-poor binary stars and in the second part in the notation [element/Fe] which might be 


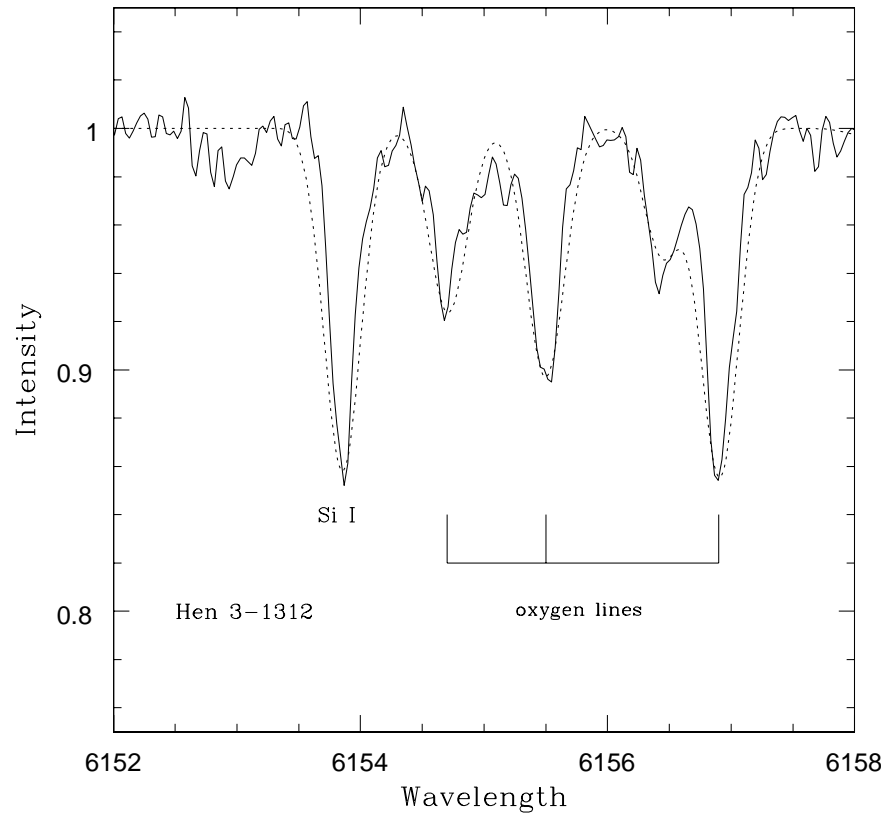

Fig. 5. Spectrum-synthesis fit to the $6152 \AA-6158 \AA$ region of Hen 3-1312. Thick line represesnts the observed spectrum and the dotted line is a synthetic spectrum calculated for $\log \epsilon(\mathrm{O})=8.63$, $T_{\text {eff }}=6500 \mathrm{~K}$ and $\log g=0.8$.

better to compare Hen 3-1312 with other post-AGB stars. As Table 6 shows, Group A stars are the same as metal-poor binary post-AGB stars. The other stars (Group B), individually named, are other post-AGB stars.

As Table 6 indicates, Population I supergiants show evidence of hydrogen-burning products at their surfaces: nitrogen is overabundant relative to Sun and carbon and oxygen are underabundant; solar values for $\log \epsilon$ (element) for carbon, nitrogen and oxygen are respectively, 8.52, 7.92 and 8.83 (Grevesse $\&$ Sauval 1998). The $21 \mu \mathrm{m}$ stars display a different pattern. They show direct evidence of the association of s-process enhancements with shell-flashes and dredge-up and in fact, these stars are those that have highest carbon overabundance and highest $<[\mathrm{s} / \mathrm{H}]>$ (van Winckel \& Reyniers 2000). The mean $\alpha$ and the heavy elements for Population I supergiants is solar (see Fig. 12 of Luck \& Bond 1989) while the overabundances, to iron, in the $21 \mu \mathrm{m}$ stars may reflect the chemical history of the Galaxy (van Winckel \& Reyners 2000). Sulfur in Population I supergiants and in $21 \mu \mathrm{m}$ stars is so$\operatorname{lar}\left(\log \epsilon^{\odot}(S)=7.33\right)$ and is $2-5$ times underabundant with respect to the Sun in Hen 3-1312, in Group A stars and also in the other post-AGB objects.

It seems, from Table 6 , that the abundance pattern of Hen 3-1312 could be related to the other post-AGB stars that do not belong to any group of the stars listed in the first part of Table 6, i.e., Population I supergiants, $21 \mu$ stars and the metal-poor binary post-AGB stars. There are four stars in Table 6 that display similar [element $/ \mathrm{Fe}]$ and $[\alpha / \mathrm{Fe}]$ ratios seen in Hen 3-1312; HD 161796, HR 6144, SAO 239853 and HD 133656 except for the ratio [s/Fe]. All these four stars have modest carbon, oxygen and nitrogen overabundances and equal $[\alpha / \mathrm{Fe}]$ ratios. However with the exception of HR 6144, they do not show enhancements of elements created by slow neutron

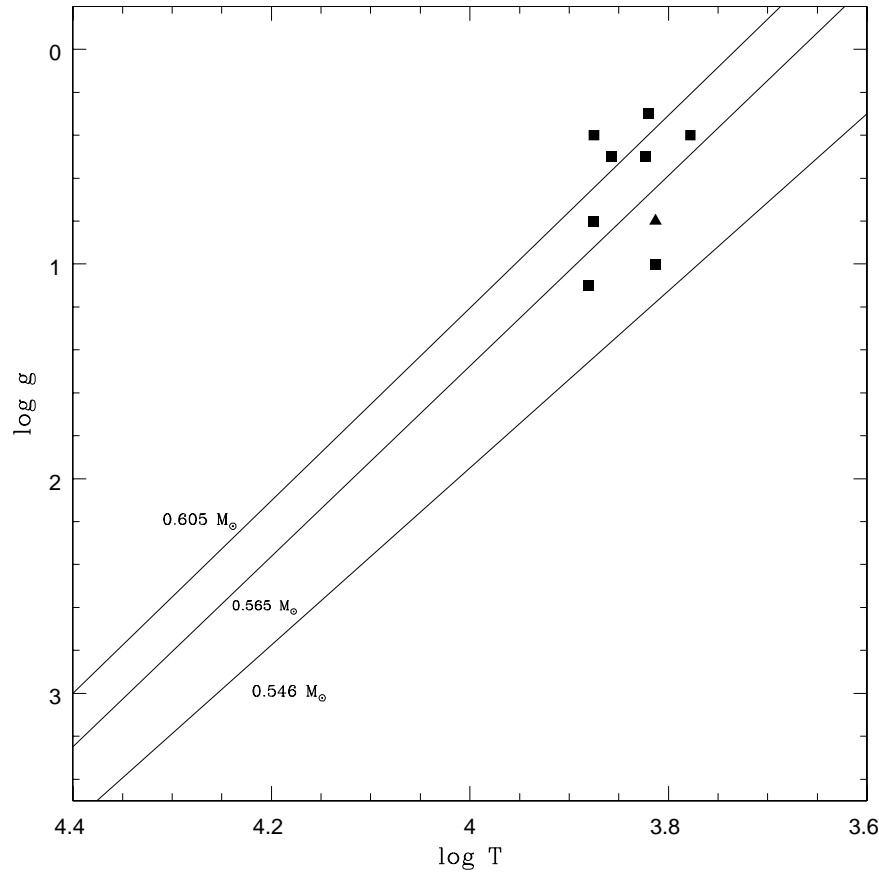

Fig. 6. Logarithmic surface gravity versus effective temperature diagram showing location of Hen 3-1312 (filled triangle) and previously identified post-AGB objects (filled squares). Data from postAGB stars were taken from Table 1 of Luck (1993). Post-HB tracks of Schönberner (1983) and Blöcker \& Schönberner (1990) are also shown.

capture reactions. But even in HR 6144, the enhancement of the elements created by s-process nucleosynthesis is not strong and could be regarded as a scatter in the chemical history of the Galaxy. The observed ratio [s/Fe] in Hen 3-1312 should be considered with some caution since is based only on one barium line. No other lines such as zirconium, yttrium or neodymium were detected in Hen 3-1312.

Luck (1993) consider the possibility that post-AGB stars that are not s-process enriched would be descendents of the $J$-type carbon stars. $J$-type carbon stars are obviously enriched in carbon, but the s-process elements have near-normal abundances (Abia \& Isern 2000). This could explain the low $[\mathrm{s} / \mathrm{Fe}]$ ratio seen in some of the post-AGB stars listed in Table 6. Similar $[\mathrm{C} / \mathrm{Fe}]$ in Table 6 , were obtained for the sample stars analyzed by Abia \& Isern (2000). If indeed Hen 3-1312 is a member of the same class of post-AGB stars that are not sprocess enriched it remains difficult to explain its low $[\mathrm{C} / \mathrm{Fe}]$ ratio as well as its relatively high $[\mathrm{s} / \mathrm{Fe}]$ ratio.

Hen 3-1312 has almost the same abundance pattern as ROA 24, a post-AGB star candidate in $\omega$ Centauri, however with a smaller $[\mathrm{C} / \mathrm{Fe}]$ ratio and a higher $[\mathrm{s} / \mathrm{Fe}]$ ratio. Gonzalez \& Wallerstein (1992) concluded that ROA 24 may be a postAGB star or a star undergoing a blue loop on the upper Asymptotic Giant Branch. However it may be tempting to apply the same nature to Hen 3-1312, due to similar observed abundances, the ratio [s/Fe] in Hen 3-1312 is based on one barium line while in ROA 24 is based in seven elements. In addition carbon abundance as well as its metallicity in ROA 24 is respectively, higher and lower than Hen 3-1312. 
Table 5. Other absorption lines studied and their respective abundance.

\begin{tabular}{|c|c|c|c|c|c|c|c|}
\hline$\lambda(\AA)$ & Species & $\chi(\mathrm{eV})$ & $\log g f$ & Ref. & $\mathrm{W}_{\lambda}(\mathrm{m} \AA)$ & $\log \epsilon$ & {$[\mathrm{X} / \mathrm{H}]$} \\
\hline 5380.310 & $\mathrm{CI}_{\mathrm{I}}$ & 7.68 & -0.78 & LRB & 41 & 7.35 & \\
\hline 7111.480 & C I & 8.64 & -1.32 & LRB & 30 & 7.70 & \\
\hline 7113.180 & C I & 8.64 & -0.95 & LRB & 42 & 7.51 & \\
\hline 7115.190 & C I & 8.64 & -0.89 & LRB & 34 & 7.35 & \\
\hline 7116.990 & $\mathrm{CI}_{\mathrm{I}}$ & 8.64 & -1.08 & LRB & 39 & 7.61 & \\
\hline \multirow[t]{2}{*}{7119.660} & CI & 8.64 & -1.31 & LRB & 20 & 7.46 & \\
\hline & & & & & & $\begin{array}{c}7.50 \\
\pm 0.14\end{array}$ & -1.02 \\
\hline 8683.400 & $\mathrm{NI}_{\mathrm{I}}$ & 10.3 & 0.11 & LL85 & 144 & 8.50 & \\
\hline 8686.150 & N I & 10.3 & -0.35 & LL85 & 100 & 8.43 & \\
\hline \multirow[t]{2}{*}{8711.700} & N I & 10.3 & -0.18 & LL85 & 98 & 8.24 & \\
\hline & & & & & & $\begin{array}{c}8.39 \\
\pm 0.13\end{array}$ & +0.47 \\
\hline 6160.800 & $\mathrm{Na} \mathrm{I}$ & 2.10 & -1.23 & LL78 & 10 & 5.61 & -0.72 \\
\hline 8717.833 & $\mathrm{Mg}_{\mathrm{I}}$ & 5.91 & -0.71 & WSM & 47 & 7.09 & \\
\hline \multirow[t]{2}{*}{8736.040} & $\mathrm{Mg}_{\mathrm{I}}$ & 5.94 & -0.34 & WSM & 53 & 6.83 & \\
\hline & & & & & & 6.96 & -0.62 \\
\hline 5665.600 & Si I & 4.92 & -1.11 & LB85 & 15 & 7.28 & \\
\hline 5708.440 & Si I & 4.95 & -1.49 & LB85 & 34 & 7.11 & \\
\hline \multirow[t]{3}{*}{5948.540} & Si I & 5.08 & -1.22 & LB85 & 50 & 7.20 & \\
\hline & & & & & & 7.19 & -0.35 \\
\hline & & & & & & \pm 0.09 & \\
\hline 8693.960 & S I & 7.87 & -0.41 & LL85 & 38 & 6.60 & \\
\hline \multirow[t]{2}{*}{8694.640} & S I & 7.87 & 0.06 & LL85 & 91 & 6.84 & \\
\hline & & & & & & 6.67 & -0.66 \\
\hline 5581.970 & $\mathrm{Ca} \mathrm{I}$ & 2.52 & -0.63 & LB85 & 32 & 5.55 & \\
\hline 5588.760 & $\mathrm{CaI}$ & 2.53 & -0.05 & LB85 & 89 & 5.78 & \\
\hline 5590.120 & $\mathrm{Ca} \mathrm{I}$ & 2.52 & -0.74 & LB85 & 25 & 5.52 & \\
\hline 5594.470 & $\mathrm{CaI}$ & 2.52 & -0.31 & LB85 & 73 & 5.82 & \\
\hline 5601.280 & $\mathrm{Ca} \mathrm{I}$ & 2.53 & -0.63 & LB85 & 34 & 5.50 & \\
\hline 6102.720 & $\mathrm{Ca} \mathrm{I}$ & 1.88 & -0.79 & LB85 & 57 & 5.53 & \\
\hline 6122.220 & $\mathrm{Ca} \mathrm{I}$ & 1.89 & -0.19 & LB85 & 117 & 5.78 & \\
\hline \multirow[t]{2}{*}{6493.780} & $\mathrm{Ca} \mathrm{I}$ & 2.52 & -0.39 & LB85 & 62 & 5.66 & \\
\hline & & & & & & $\begin{array}{c}5.67 \\
\pm 0.13\end{array}$ & -0.67 \\
\hline 4810.530 & $\mathrm{Zn} \mathrm{I}$ & 4.06 & -0.39 & BG80 & 43 & 3.86 & \\
\hline \multirow[t]{2}{*}{4810.530} & $\mathrm{Zn} \mathrm{I}$ & 4.06 & -0.17 & BG80 & 47 & 3.74 & \\
\hline & & & & & & 3.80 & -0.80 \\
\hline 6496.900 & Ba II & 0.60 & -0.38 & WM80 & 161 & 1.88 & -0.25 \\
\hline
\end{tabular}

References to Table 5:

BG80: Biémont \& Godefroid (1980); LB85: Luck \& Bond (1985); LRB: Lambert et al. (1982); LL85: Luck \& Lambert (1985); LL78: Lambert \& Luck (1978); WM80: Wiese \& Martin (1980).

From what was said above it is difficult to fit Hen 3-1312 into one or another category of some post-AGB stars already known since its abundance pattern does not exhibit a clear trend. A study in order to obtain the orbital parameters would put some constraints on the evolution in this binary system and also on how the mass-transfer may have happened in the past.

\section{Conclusions}

The low-resolution spectrum of Hen 3-1312 indicates that Hen 3-1312 is a binary system, as can be judged from the fact that a hot source should be present in order to account for the observed emission-lines seen over a continuum of F-type star. 
Table 6. Mean abudances and metallicities of Population I supergiants, post-AGB stars and Hen 3-1312. [element/Fe] ratio for other post-AGB stars is also shown.

\begin{tabular}{|c|c|c|c|c|c|c|c|c|c|}
\hline Star & {$[\mathrm{Fe} / \mathrm{H}]^{1}$} & $<\log \epsilon(\mathrm{C})>$ & $<\log \epsilon(\mathrm{N})>$ & $<\log \epsilon(\mathrm{O})>$ & $<\log \epsilon(\mathrm{S})>$ & $<[\alpha / \mathrm{H}]>^{2}$ & $<[\mathrm{s} / \mathrm{H}]>^{3}$ & {$[\mathrm{Zn} / \mathrm{H}]$} & reference \\
\hline Hen 3-1312 & -1.1 & 7.51 & 8.39 & 8.63 & 6.67 & -0.55 & -0.25 & -0.25 & 1 \\
\hline Pop I sg & +0.13 & 8.16 & 8.48 & 8.57 & 7.33 & +0.23 & +0.18 & - & 2 \\
\hline $21 \mu \mathrm{m}$ & -0.3 to -1.0 & 8.69 & 7.82 & 8.57 & 7.29 & -0.37 & +0.78 & - & 3 \\
\hline \multirow[t]{2}{*}{ Group A } & -1.5 to -4.5 & 8.22 & 8.01 & 8.51 & 6.93 & -2.11 & - & -1.3 & 4 \\
\hline & & {$[\mathrm{C} / \mathrm{Fe}]$} & {$[\mathrm{N} / \mathrm{Fe}]$} & {$[\mathrm{O} / \mathrm{Fe}]$} & {$[\mathrm{S} / \mathrm{Fe}]$} & {$[\alpha / \mathrm{Fe}]$} & {$[\mathrm{s} / \mathrm{Fe}]^{3}$} & & \\
\hline Hen 3-1312 & -1.1 & +0.1 & +1.6 & +0.9 & +0.4 & +0.6 & +0.8 & & 1 \\
\hline HD 161796 & -0.3 & +0.3 & +1.1 & +0.4 & +0.7 & +0.4 & 0.0 & & 5 \\
\hline HR 6144 & -0.4 & +0.3 & +0.9 & +0.3 & +0.4 & +0.5 & +0.2 & & 5 \\
\hline HR 7671 & -1.1 & -0.3 & +0.1 & -0.3 & +0.2 & +0.4 & +0.6 & & 5 \\
\hline 89 Her & -0.4 & +0.3 & +0.6 & +0.1 & +0.1 & +0.2 & 0.0 & & 5 \\
\hline SAO 173329 & -0.8 & +0.3 & +0.3 & - & +0.1 & +0.1 & - & & 6 \\
\hline HD 95767 & +0.1 & -0.1 & -0.2 & -0.5 & -0.1 & 0.0 & - & & 6 \\
\hline SAO 239853 & -0.8 & +0.4 & +0.6 & +0.8 & +0.4 & +0.4 & -0.4 & & 6 \\
\hline HD 107369 & -1.1 & $\leq-0.2$ & +0.4 & 0.0 & +0.1 & +0.2 & -0.1 & & 6 \\
\hline HD 108015 & -0.1 & +0.1 & +0.2 & -0.1 & -0.1 & +0.1 & - & & 6 \\
\hline HD 131356 & -0.6 & +0.3 & +0.3 & -0.1 & +0.1 & 0.0 & - & & 6 \\
\hline HD 133656 & -0.7 & +0.2 & +0.5 & +0.6 & +0.4 & +0.2 & -0.4 & & 6 \\
\hline ROA 24 & -2.1 & +0.6 & +1.8 & +1.3 & +0.7 & +0.5 & +0.3 & & 7 \\
\hline
\end{tabular}

Notes to Table 6:

1: mean value for Pop I sg.

2: $\alpha=\mathrm{Mg}, \mathrm{Si}, \mathrm{Ca}$ and $\mathrm{Al}$ for Pop I sg.

$\alpha=$ Table 4 of van Winckel \& Reyniers (2000) for $21 \mu \mathrm{m}$ stars.

$\alpha=\mathrm{Mg}, \mathrm{Si}$ and Ca for stars of Ref. 4 and Hen 3-1312.

$\alpha=\mathrm{Mg}$, Si and $\mathrm{Al}$ for Group A stars.

$\alpha=\mathrm{Mg}, \mathrm{Si}, \mathrm{Ca}$ and Ti for stars of Ref. 5 .

3: $\mathrm{s}=\mathrm{Y}, \mathrm{Zr}, \mathrm{Ba}, \mathrm{La}, \mathrm{Ce}$ and $\mathrm{Nd}$ for Pop I sg.

$s=$ Table 4 of van Winckel \& Reyniers (2000) for $21 \mu \mathrm{m}$ stars.

$s=$ Table 8 of Luck et al. (1990) (Ref. 5).

$s=$ Ba for Hen 3-1312.

$s=$ Table 11 of van Winckel (1997).

Refs.:

1: This work.

2: Luck \& Bond (1989); Luck \& Lambert (1985).

3: van Winckel \& Reyniers (2000).

4: Trams et al. (1993).

5: Luck et al. (1990).

6: van Winckel (1997).

7: Gonzalez \& Wallerstein (1994).

Analysis of the high-resolution spectrum placed this star in the $\log g$ - $\log T$ plane together with other post-AGB stars. The nature of the nebulae is a matter of debate. In the $D^{\prime}$-type symbiotics, for example, the nebulae is the ionized fossil AGB remnant planetary-nebulae of the hot star in the system (Corradi \& Schwarz 1997). In the $D^{\prime}$-type symbiotics the nebulae is older than those in the $D$-type symbiotics (systems containing a Mira as cool star). In Hen 3-1312 the age of the nebulae could be even older than in $D^{\prime}$-types symbiotics (Corradi \& Schwartz 1997; Schwartz 1991).

Based on the analysis of the high resolution spectrum it was adopted the following atmospheric parameters for Hen 3-1312: $T_{\text {eff }}=6500 \mathrm{~K}, \log g=0.8, V_{\mathrm{t}}=2.6 \mathrm{~km} \mathrm{~s}^{-1}$ and $[\mathrm{Fe} / \mathrm{H}]=-1.1$. The radial velocity, $V_{\mathrm{r}}=-77 \mathrm{~km} \mathrm{~s}^{-1}$ and with a distance of the Galactic plane $(\approx 500 \mathrm{pc})$, Hen 3-1312 maybe considered to belong to the old disc population. The study of chemical composition shows that carbon, oxygen, nitrogen and sulfur in Hen 3-1312 is basically solar but the $\alpha$-elements such as magnesium, silicon and calcium are underabundant with respect to the Sun. The evolutionary status of Hen 3-1312 is not very clear since its abundance pattern does not fit in any of the post-AGB stars analyzed so far.

\section{References}

Abia, C., \& Isern, J. 2000, ApJ, 536, 438

Baldwin, J. A., Phillips, M., \& Terlevich, R. 1981, PASP, 93, 5

Beaulieu, S. F., Dopita, M. A., \& Freeman, K. C. 1999, ApJ, 515, 610 
Biémont, E., \& Godefroid, M. 1980, Phys. Scr., 22, 231

Blöcker, T., \& Schönberner, D. 1990, A\&A, 240, L11

Corradi, R. L. M., \& Schwarz, H. 1997, Extended optical nebulae around symbiotic stars, ed. J. Mikolajewska, in Physical Processes in Symbiotic Binaries, 147

de Freitas Pacheco, J. A., \& Veliz, J. A. 1987, Rev. Mex. Astron. Astrof., 15, 89

Gonzalez, G., \& Wallerstein, G. 1992, MNRAS, 254, 343

Grevesse, N., \& Sauval, A. J. 1998, Spa. Sci. Rev., 85, 161

Hamuy, M., Suntzeff, N. B., Heathcote, S. R., et al. 1994, PASP, 106, 566

Hummer, D. G., \& Storey, P. J. 1987, MNRAS, 224, 801

Kaufer, A., Stahl, O., Tubbesing, S., et al. 1999, The Mesenger, 95, 8

Kodaira, K., Greenstein, J. L., \& Oke, J. B. 1970, ApJ, 159, 485

Kohoutek, L. 1978, in New and Misclassified Planetary Nebulae, IAU Symp., 76, 47 (Dordrecht: D. Reidel Publishing Co.)

Kurucz, R. L. 1993, CD-ROM 13, Atlas9 Stellar Atmosphere Programs and $2 \mathrm{~km} \mathrm{~s}^{-1}$ Grid (Cambridge: Smithosian Astrophys. Obs.)

Lambert, D. L., \& Luck, R. E. 1978, MNRAS, 183, 79

Lambert, D. L., Roby, S. W., \& Bell, R. A. 1982, ApJ, 254, 664

Lambert, D. L., Heath, J. E., Lemke, M., \& Drake, J. 1996, ApJS, 103, 183

Lamers, H. J. L. M., Zickgraf, F.-J., de Winter, D., Houziaux, L., \& Zorec, J. 1998, A\&A, 340, 117

Luck, R. E., \& Bond, H. 1985, ApJ, 292, 559

Luck, R. E., \& Lambert, D. 1985, ApJ, 298, 782
Luck, R. E., \& Bond, H. 1989, ApJS, 71, 559

Luck, R. E., Bond, H., \& Lambert, D. L. 1990, 357, 188

Luck, R. E. 1993, The chemical composition of luminous high-latitude stars post-AGB stars, ed. D. D. Sasselov, in Luminous HighLatitude Stars, 87

Lutz, J. H., \& Kaler, J. B. 1983, PASP, 95, 739

Oke, J. B. 1974, ApJS, 27, 21

Osterbrock, D. E. 1974, Astrophysics of Gaseous Nebulae (San Francisco: W.H. Freeman)

Parthasarathy, M., Vijapurkar, J., \& Drilling, J. S. 2000, A\&AS, 145, 269

Phillips, J. P. 2001, A\&A, 367, 927

Sanduleak, N., \& Stephenson, C. B. 1972, PASP, 84, 816

Schönberner, D. 1983, A\&A, ApJ, 272, 708

Schmeja, S., \& Kimeswenger, S. 2002, Rev. Mex. Astron. Astrof., 12, 176

Schwarz, H. 1991, A\&A, 243, 469

Sneden, C. 1973, Ph.D. Thesis, Univ. of Texas

Trams, N. R., Waelkens, C., \& Waters, L. B. F. M. 1993, Extremely metal-poor post-AGB stars, in Luminous High-Latitude Stars, ed. D. D. Sasselov, 103

van Winckel, H. 1997, A\&A, 319, 561

van Winckel, H., \& Reyniers, M. 2000, A\&A, 354, 135

Venn, K. A. 1993, ApJ, 414, 316

Wiese, W. L., \& Martin, G. A. 1980, NSDRS-NBS, 68 


\section{Online Material}


C. B. Pereira: The nature of Hen 3-1312: A post-AGB star in a binary system, Online Material p 2

Table 4. Observed Fe I and Fe II absorption lines in Hen 3-1312.

\begin{tabular}{|c|c|c|c|c|}
\hline Element & $\lambda(\AA)$ & $\chi(\mathrm{eV})$ & $\log g f$ & $W_{\lambda}(\mathrm{m} \AA)$ \\
\hline \multirow[t]{47}{*}{ Fe I ...... } & 5171.60 & 1.49 & -1.76 & 107 \\
\hline & 5194.94 & 1.56 & -2.06 & 70 \\
\hline & 5198.71 & 2.22 & -2.14 & 36 \\
\hline & 5202.34 & 2.18 & -1.84 & 71 \\
\hline & 5216.27 & 1.61 & -2.12 & 63 \\
\hline & 5232.94 & 2.94 & -0.08 & 125 \\
\hline & 5281.79 & 3.04 & -0.83 & 54 \\
\hline & 5302.31 & 3.28 & -0.74 & 58 \\
\hline & 5324.18 & 3.21 & -0.10 & 117 \\
\hline & 5339.93 & 3.27 & -0.68 & 60 \\
\hline & 5341.02 & 1.61 & -1.95 & 88 \\
\hline & 5353.37 & 4.10 & -0.68 & 24 \\
\hline & 5364.88 & 4.45 & 0.23 & 50 \\
\hline & 5367.47 & 4.42 & 0.44 & 66 \\
\hline & 5369.96 & 4.37 & 0.54 & 76 \\
\hline & 5383.37 & 4.31 & 0.65 & 83 \\
\hline & 5389.48 & 4.42 & -0.25 & 27 \\
\hline & 5393.17 & 3.24 & -0.72 & 53 \\
\hline & 5400.50 & 4.37 & -0.10 & 38 \\
\hline & 5405.77 & 0.99 & -1.85 & 137 \\
\hline & 5410.91 & 4.47 & 0.40 & 63 \\
\hline & 5424.07 & 4.32 & 0.58 & 97 \\
\hline & 5434.52 & 1.01 & -2.12 & 120 \\
\hline & 5487.75 & 4.32 & -0.65 & 27 \\
\hline & 5497.52 & 1.01 & -2.84 & 54 \\
\hline & 5506.78 & 0.99 & -2.80 & 59 \\
\hline & 5554.90 & 4.55 & -0.38 & 20 \\
\hline & 5563.60 & 4.19 & -0.84 & 16 \\
\hline & 5569.62 & 3.42 & -0.49 & 65 \\
\hline & 5572.84 & 3.40 & -0.28 & 73 \\
\hline & 5576.09 & 3.43 & -0.85 & 42 \\
\hline & 5586.76 & 3.37 & -0.12 & 78 \\
\hline & 5615.64 & 3.33 & 0.00 & 111 \\
\hline & 5762.99 & 4.21 & -0.41 & 35 \\
\hline & 6020.17 & 4.61 & -0.21 & 32 \\
\hline & 6024.06 & 4.55 & -0.06 & 42 \\
\hline & 6136.61 & 2.45 & -1.40 & 55 \\
\hline & 6137.69 & 2.59 & -1.40 & 48 \\
\hline & 6230.72 & 2.56 & -1.28 & 64 \\
\hline & 6252.56 & 2.40 & -1.72 & 40 \\
\hline & 6254.26 & 2.28 & -1.72 & 40 \\
\hline & 6393.60 & 2.42 & -1.43 & 48 \\
\hline & 6411.65 & 3.65 & -0.66 & 43 \\
\hline & 6419.95 & 4.73 & -0.09 & 23 \\
\hline & 6421.35 & 2.28 & -2.01 & 40 \\
\hline & 6430.85 & 2.18 & -2.01 & 39 \\
\hline & 6592.91 & 2.73 & -1.47 & 37 \\
\hline \multirow[t]{12}{*}{ Fe II ..... } & 5132.66 & 2.81 & -4.00 & 40 \\
\hline & 5284.10 & 2.89 & -3.01 & 115 \\
\hline & 5325.56 & 3.22 & -3.17 & 91 \\
\hline & 5414.05 & 3.22 & -3.62 & 42 \\
\hline & 5425.25 & 3.20 & -3.21 & 81 \\
\hline & 5991.37 & 3.15 & -3.56 & 48 \\
\hline & 6084.09 & 3.20 & -3.80 & 32 \\
\hline & 6149.25 & 3.89 & -2.72 & 82 \\
\hline & 6247.55 & 3.89 & -2.34 & 134 \\
\hline & 6369.46 & 2.89 & -4.19 & 38 \\
\hline & 6416.92 & 3.89 & -2.68 & 64 \\
\hline & 6432.68 & 2.89 & -3.58 & 68 \\
\hline
\end{tabular}

